\title{
LOCALIZAÇÃO ESPACIAL DO SEGMENTO DA INDUSTRIA DE PRODUTOS MINERAIS NÃO METÁLICOS NO RIO GRANDE DO NORTE (1990 - 2010)
}

\author{
SPACE LOCALIZATION OF NON-METALLIC MINERAL PRODUCTS SEGMENT IN \\ RIO GRANDE DO NORTE (1990 - 2010)
}

\author{
Osmar Faustino OLIVEIRA ${ }^{1}$ \\ Manoel Raymundo de CARVALHO NETO2 \\ Pollyanna Neves de MEDEIROS ${ }^{3}$ \\ Flávio Antônio Miranda de SOUZA ${ }^{4}$
}

Artigo recebido em 12/05/2019 e aceito em 31/07/2019

Palavras-chave:

Localização,

Emprego,

Rio Grande do Norte.

\begin{abstract}
R E S U M O
0 reconhecimento dos aglomerados produtivos no processo de crescimento econômico é um tema que vem sendo estudado na literatura de economia regional nos últimos anos. 0 problema da pesquisa, materializado neste estudo, consiste em identificar e analisar as aglomerações produtivas no setor de produtos minerais não metálicos no estado do Rio Grande do Norte. 0 objetivo principal deste estudo é identificar e analisar a evolução das aglomerações produtivas do setor da indústria de produtos não metálicos no estado do Rio Grande do Norte nos anos 1990, 2000 e 2010. Realizou-se uma revisão bibliográfica que deu suporte à pesquisa, e um levantamento de dados secundários, que deram suporte ao cálculo do índice de Hoover e foram obtidos a partir da Relação Anual de Informações Sociais (RAIS), disponibilizados pelo Ministério do Trabalho e Emprego (MTE). Uma vez coletados os dados da RAIS, foi preciso buscar a classificação de Subsetores segundo a classificação do IBGE, mais especificamente aos 15 subsetores que compõem o setor industrial. Os resultados mostraram que no ano de 1990 a 2010 surgiram, 44 novas aglomerações.
\end{abstract}

\begin{abstract}
A B S T RA C T
The recognition of productive clusters in the process of economic growth is an issue that has been studied in the literature on regional economics in recent years. The research problem, materialized in this study, is to identify and analyze productive agglomerations in the sector of nonmetallic mineral products in the state of Rio Grande do Norte. The main objective of this study is to identify and analyze the evolution of the productive agglomerations of the sector of non-metallic products industry in the state of Rio Grande do Norte in the years 1990, 2000 and 2010. A bibliographical review was carried out that supported the research, and a survey of secondary data, which supported the calculation of the Hoover index and were obtained from the Annual Social Information Report (RAIS), made available by the Ministry of Labor and Employment (MTE). Once the RAIS data were collected, it was necessary to seek the classification of Sub-Sectors according to the IBGE classification, more specifically to the 15 subsectors that make up
\end{abstract}

\footnotetext{
1 Mestrando em Desenvolvimento Urbano pela Universidade Federal de Pernambuco. Especialista em Mercado de Capitais pela Universidade Federal do Rio Grande do Norte. E-mail: osmarfaustino@yahoo.com.br.

2 Graduação em Administração com Habilitação em Marketing pela Escola Superior de Marketing (ESM/FAMA). Mestre em Gestão e Economia da Saúde pela Universidade Federal de Pernambuco (UFPE). Secretário no Programa de Pós-Graduação em Gerontologia do Centro de Ciências da Saúde da UFPE. E-mail: manoelneto.ufpe@gmail.com.

3 Mestranda no Programa de Pós-Graduação em Economia Regional da UFRN. Graduada em Ciências Econômicas pela Universidade Federal do Rio Grande do Norte. E-mail: pollyannanmedeiros@outlook.com.

4 Professor Titular do Departamento de Expressão Gráfica e Professor permanente do Programa de Pós-graduação em Desenvolvimento Urbano (MDU) da UFPE. E-mail: fdesouza67@gmail.com.
} 
the industrial sector. The results showed that in the year 1990 to 2010,44 new agglomerations appeared.

\section{INTRODUÇÃO}

O debate acerca do conceito de cluster no Brasil, assim como no plano internacional, tem movido o interesse de estudiosos e gestores de políticas públicas, desde que a prosperidade de aglomerações setoriais de pequenas e médias empresas em plena crise fordista, no início dos anos 1980, gerou revisitações às contribuições de Alfred Marshall sobre os distritos industriais. Incentivada pelos manifestos escritos de Porter $(1990,1998)$ sobre competitividade de firmas e regiões, e pelo prestígio por parte do mainstream do papel dos rendimentos crescentes para a dinâmica econômica nas chamadas novas teorias de crescimento e comércio internacional (Krugman, 1995), a importância da localização e a noção de cluster têm-se conquistado tanto no campo acadêmico quanto no de formulação de política industrial em países de variada formação econômica (FERNANDES; LIMA, 2006).

O cluster, segundo Lopes Neto (1998), são várias indústrias ou empresas relacionadas, todas bem-sucedidas no mesmo local. O cluster é inerente a toda economia e indústria e oferece vários benefícios competitivos para grandes e pequenas empresas. Segundo o referente autor o cluster é uma rede de funções conectadas, são atividades que tem ligação numa cadeia industrial, ou seja, é uma aglomeração geográfica de diferentes atividades e que no caso do referente trabalho, nos diversos segmentos da indústria. Como definido por alguns economistas, aglomeração econômica, transmite uma união de esforços entre os governos, empresários, agentes financeiros e outros segmentos representativos da sociedade, com o objetivo de levar maior desenvolvimento para o Nordeste, sendo assim um tema bastante discutido na economia regional que enfatiza as políticas de desenvolvimento para o Nordeste brasileiro.

O problema da pesquisa, materializado neste estudo, consiste em identificar e analisar as aglomerações produtivas no setor de produtos minerais não metálicos no estado do Rio Grande do Norte, tendo como objetivo principal a identificação e análises da evolução das aglomerações produtivas deste setor identificando o emprego formal.

As políticas regionais têm o objetivo principal de criar nas regiões um ambiente favorável para atração de investimentos, desenvolvendo e fortalecendo as instituições para conduzir ao aumento de sua capacidade de transformação e de aceitação de inovações tecnológicas que favoreçam maior integração e coesão espacial dentro da região e entre as demais regiões do país.

Nesse contexto o presente estudo procura mostrar a localização dos empregos formais nos segmentos da indústria nos estados da Paraíba e do Rio Grande do Norte nos anos 1990, 2000 e 2010, 
sendo o trabalho estruturado da seguinte forma: referencial teórico e metodologia, considerações finais e referências.

\section{REFERENCIAL TEÓRICO E METODOLOGIA}

Acadêmicos, políticos e empresários discutem sobre a forma sucedida de crescimento rápido das empresas, evidenciando as configurações de aprendizagem e os avanços no estoque de conhecimento das firmas. Os indivíduos e instituições precisam renovar suas competências, devido às rápidas mudanças que ocorrem constantemente no ambiente socioeconômico, ao mesmo tempo em que vários segmentos da sociedade são afetados pela aceleração de transformações. 0 conhecimento inicia-se em um ambiente comum e intensifica-se com a produção globalizada. Esse processo se propaga em um território localizado, que permite desencadear o desenvolvimento das firmas, aliadas às particularidades que irão contribuir para a produção setorial. A transmissão do conhecimento ocorre entre pessoas, firmas, organizações (RODRIGUES; et. al.; 2012).

A firma não é considerada um agente isolado no processo de inovação, mas parte de um ambiente com capacidade inovativa. Esse conjunto de elementos e relacionamentos é representado por vínculos entre firmas, clientes, organizações de pesquisa, sistema educacional e demais autoridades locais que interagem de forma cooperativa (LASTRES \& CASSIOLATO 2005). A partir desse ambiente inovativo é correto pensar em território localizado, no qual a cultura e o conjunto de normas praticadas constantemente são suficientes para a construção de um espaço social que influencie as interações localizadas. Assim, fizeram-se necessários estudos que abordassem as relações interfirmas, ou seja, as aglomerações produtivas locais, cuja proximidade territorial entre os agentes (organizações públicas e privadas ou empresas) se constitui no elemento determinante de transmissão de conhecimento.

Diante desse contexto territorial, difundiram-se novas vertentes de estudos que analisam as relações interfirmas em ambientes localizados, denominados como sistemas produtivos locais. Em seus estudos, Schmitz \& Nadvi (1999) definiram o sistema produtivo ou cluster como uma concentração espacial de firmas em que, conforme abordado por Suzigan et al. (2004), a interdependência, articulação e vínculo resultam em aprendizagem, cooperação e interação, com potencial de gerar o incremento da capacidade de inovação endógena, da competitividade e do desenvolvimento local (RODRIGUES; et. Al.; 2012).

A década de 1990 caracteriza-se pela inserção do Brasil no processo de mundialização do capital. A abertura comercial ampliou o nível de competividade da produção brasileira para com as empresas internacionais. No Brasil, a reestruturação produtiva implicou um processo de descentralização produtiva de alguns setores importantes, principalmente os intensivos em trabalho. 
A descentralização produtiva caracterizou-se muito mais por ser uma relocalização industrial do que, em sua essência, descentralização de capitais (PEREIRA, 2008).

O reconhecimento dos aglomerados produtivos no processo de crescimento econômico é um tema que vem sendo estudado na literatura de economia regional nos últimos anos. Este tem sido analisado devido à organização interfirmas, a qual permite aos agentes econômicos responderem de forma eficaz aos desafios impostos pela dinâmica econômica. Estas interações estão correlacionadas à complementaridade entre as estruturas produtivas, inovações tecnológicas, aquisição e fusão dos conhecimentos, e acabam viabilizando a competição e a formação de novas tecnologias. No entanto, os métodos que vêm sendo utilizados não contemplam as proximidades geográficas, deixando de considerar os impactos sobre a organização espacial das firmas. A identificação das aglomerações produtivas pode ser subestimada, se os municípios possuírem relações espaciais. Estudos que consideram apenas indicadores de concentração, como Quociente Locacional (QL) segundo (RODRIGUES; et. Al.; 2012).

Na maioria dos países a atividade da indústria de produtos minerais não metálicos se organiza em forma de aglomerações produtivas, aproveitando-se das muitas externalidades geradas que se constituem em fontes de seu dinamismo.

O cluster é inerente a toda economia e indústria e oferece vários benefícios competitivos para grandes e pequenas empresas. Definido por alguns economistas como aglomerações econômicas, eles transmitem uma união de esforços entre os governos, empresários, agentes financeiros e outros segmentos representativos da sociedade, com o objetivo de levar maior desenvolvimento para o Nordeste (LOPES NETO, 1998).

Correlacionando com o parágrafo acima, pode-se dizer que, cluster significa a concentração espacial de atividades econômicas setorialmente especializadas que realizam intenso comércio entre si, sentido este que, apesar de demasiadamente difuso, expressa a importância da dimensão espacial para o desenvolvimento econômico. Então, os clusters adquirem relevância porque desencadeiam efeitos externos e interação entre diferentes agentes localizados próximos uns aos outros. Além disso, esse padrão específico de produção associada ao território é reconhecido como formado preponderantemente por pequenas e médias empresas. No entanto, são características mais expressivas que têm atraído a atenção de estudiosos e governantes para o fenômeno da aglomeração produtiva gerando oportunidades para eficiência coletiva decorrentes de economias externas positivas, baixos custos de transação e ação conjunta e coordenada (FERNANDES; LIMA, 2006).

Rezende (2012) demonstra que as aglomerações de empresas espacialmente concentradas e setorialmente especializadas, têm mais chances de sucesso em um ambiente competitivo e de constantes mudanças tecnológicas se elas fazem parte de um cluster, ao invés de operarem isoladamente no mercado. 
Para melhor desenvolvimento e explicitação do conteúdo deste trabalho foi realizada uma revisão bibliográfica, que deu suporte à pesquisa, e também foram levantados dados secundários que dessem suporte ao cálculo do índice de Hoover.

Os dados secundários foram coletados na Relação Anual de Informações Sociais (RAIS) disponibilizada pelo Ministério do Trabalho e Emprego (TEM). A escolha da fonte desses dados secundários, foi realizada mediante a percepção de que as informações adquiridas a partir da RAIS. São comprovadamente uma boa fonte de informações, a respeito do mercado de trabalho formal brasileiro. Depois de coletados os dados da RAIS, analisamos estes os aplicando no contexto da classificação dos 15 subsetores que compõem o setor industrial segundo o IBGE (ver tabela 1).

Para visualizar a especialização dos segmentos industriais nos estados da Paraíba e Rio Grande do Norte utilizou-se o índice de localização de Hoover (1936), ou Quociente Locacional, tendo em vista que este é uma importante medida de especialização regional desenvolvida pela teoria econômica. Tal índice é expresso por:

$$
Q L=\frac{E_{i j}}{E_{j}} / \frac{E_{i R R}}{E_{R R}}
$$

Onde:

$E_{i j}$ É o emprego da indústria $i$ na região j;

$E_{i T}$ É o emprego total na região j;

$E_{j}$ É o emprego industrial na Região referência;

$E_{i j}$ Emprego total na região de referência.

0 coeficiente de Hoover tem como característica ser uma medida relativa, permitindo assim a comparação das concentrações dos diferentes segmentos industriais (SILVEIRA NETO, 2005). Podemos ver no coeficiente de Hoover a importância do setor i da economia para a região j, que é a região de referência. Quanto maior for o índice obtido, mais concentrada e especializada é a indústria i da região de referência j.

Adotou-se como filtro para que possa ser feito o cálculo do Quociente Locacional em determinado município, uma quantidade mínima de 10 estabelecimentos industriais e 1,0 mil empregos. E ao final do cálculo, serão considerados aglomerados econômicos, os municípios que apresentarem QL igual ou superior a 3. Os demais não serão considerados aglomerações econômicas devido à falta de significativa especialização do Quociente Locacional. 


\section{ANÁLISE DESCRITIVA DOS DADOS}

O estado do Rio grande do Norte possui uma grande especialização no setor industrial de Produtos Minerais não Metálicos. No ano 1990 a quantidade de Clusters era de 6, passando em 2010 para 17 aglomerados. Isto mostra o quanto o estado vem se concentrando nessa atividade industrial, ou seja, surgiram novos onze clusters. Outros setores industriais que merecem destaque são os da Indústria Têxtil, da Extrativa Mineral e a Indústria de Calçados, pois surgiram cinco aglomerações no primeiro segmento e quatro aglomerações nos demais citados durante o período estudado. A indústria têxtil possuía apenas 1 cluster em 1990, já em 2010 ultrapassa para 6 clusters, um saldo positivo de 5 novos clusters. Em 1990 a extrativa mineral possuía quatro clusters, e em 2010 esse número duplica para oito aglomerados econômicos. A indústria calçadista em 1990 possuía 1 cluster e em 2010 esse número ultrapassa para 5. A indústria de alimentos no ano de 1990 apresentava apenas 1 aglomerado, já em 2010 a situação muda, surgem 3 novas aglomerações, ficando assim com quatro clusters (Tabelas 1 e 2).

Tabela 1. Quantidade de aglomerados industriais que apresentaram QL igual ou superior a 3 nos municípios do estado do Rio Grande do Norte1990-2010.

\begin{tabular}{lrrr}
\hline Indústria no Rio Grande do Norte & 1990 & 2000 & 2010 \\
\hline Extrativo mineral & 4 & 6 & 8 \\
\hline Indústria de produtos minerais não metálicos & 6 & 15 & 17 \\
\hline Indústria metalúrgica & 0 & 3 & 2 \\
\hline Indústria mecânica & 2 & 3 & 4 \\
\hline Indústria do material elétrico e de comunicações & 0 & 1 & 2 \\
\hline Indústria do material de transporte & 2 & 3 & 3 \\
\hline Indústria da madeira e do mobiliário & 2 & 4 & 3 \\
\hline Indústria do papel, papelão, editorial e gráfica & 1 & 1 & 3 \\
\hline Ind. da borracha, fumo, couros, peles, similares, ind. Diversas & 2 & 1 & 3 \\
Ind. química de produtos farmacêuticos, veterinários, & & & \\
perfumaria, ... & 2 & 3 & 3 \\
\hline Indústria têxtil do vestuário e artefatos de tecidos & 2 & 4 & 6 \\
\hline Indústria de calçados & 1 & 4 \\
\hline Indústria de produtos alimentícios, bebidas e álcool etílico & 1 & 2 & 5 \\
\hline Serviços industriais de utilidade pública & 1 & 3 & 4 \\
\hline Construção civil & 0 & 2 & 3 \\
\hline Soma & 0 & 2 & 2 \\
\hline Fonte: Elaboraça própra a partir dos dados da RAIS - MTE. Banco & 24 & 53 & 68 \\
\hline
\end{tabular}

$\overline{\text { Fonte: Elaboração própria a partir dos dados da RAIS - MTE. Banco de dados do GEPETIS. }}$ 
Tabela 2. Quociente Locacional da Indústria de produtos minerais não metálicos nos municípios do RN

\begin{tabular}{|c|c|c|c|}
\hline Indústria de produtos minerais não metálicos & 1990 & 2000 & 2010 \\
\hline Acari & 0,0 & 8,8 & 14,2 \\
\hline Assú & 6,3 & 8,7 & 5,6 \\
\hline Apodi & 0,0 & 4,1 & 3,7 \\
\hline Caicó & 1,0 & 1,9 & 4,3 \\
\hline Carnaúba dos Dantas & 0,0 & 35,1 & 32,5 \\
\hline Cruzeta & 0,0 & 24,1 & 17,2 \\
\hline Currais Novos & 3,0 & 4,2 & 2,0 \\
\hline Goianinha & 0,0 & 3,9 & 3,9 \\
\hline Ipanguaçú & 24,1 & 7,7 & 4,2 \\
\hline Itajá & 0,0 & 70,3 & 42,9 \\
\hline Jardim do Seridó & 0,0 & 6,4 & 7,9 \\
\hline Macaíba & 20,2 & 0,3 & 1,6 \\
\hline Nísia Floresta & 0,0 & 4,5 & 0,2 \\
\hline Ouro Branco & 0,0 & 0,0 & 8,4 \\
\hline Parelhas & 8,9 & 22,2 & 20,3 \\
\hline Pendências & 0,0 & 7,5 & 6,3 \\
\hline Santa Cruz & 0,0 & 1,9 & 3,1 \\
\hline Santana do Seridó & 0,0 & 0,0 & 23,8 \\
\hline São Gonçalo do Amarante & 8,1 & 4,2 & 2,8 \\
\hline São Jose de Mipibú & 0,0 & 4,2 & 6,4 \\
\hline Tangará & 0,0 & 0,0 & 3,1 \\
\hline
\end{tabular}

Fonte: Elaboração própria a partir dos dados da RAIS - MTE. Banco de dados do GEPETIS.

Os municípios do Rio Grande do Norte vêm ao longo dos anos se especializando cada vez mais no setor da indústria de produtos não metálicos. O município que mais se destaca é Itajá, pois em 1990 não existia nenhuma especialização, e dez anos depois, surge uma concentração do setor de 70,3. Em 2010 o nível de especialização cai para 42,85, mas, mesmo assim este município possui um dos maiores índices de especialização no setor de produtos não metálicos. A cidade de Carnaúba dos Dantas merece destaque, pois, em 1990, assim como Itajá não possuía especialização. Em 2000 chega a uma concentração de 35,12 e de 32,48 em 2010. Merece destaque também a inclusão de Santana do Seridó em 2010 com concentração industrial de 23,78 tendo em vista que nos anos de 1990 e 2000 ela não possuía especialização (Figura 1). 
Figura 1. Localização do emprego industrial na no Rio Grande do Norte em 1990

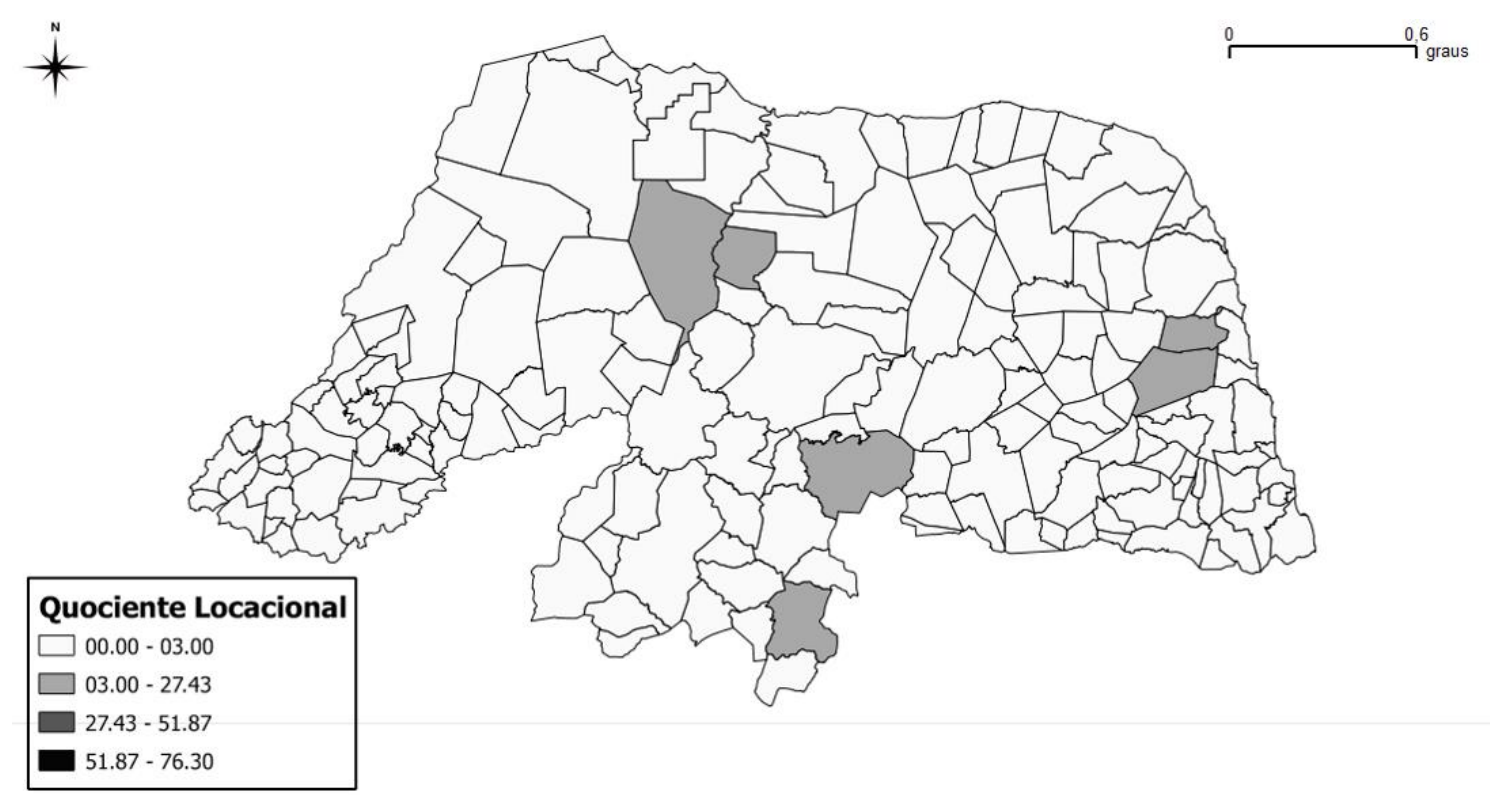

Fonte: Elaboração própria a partir dos dados da RAIS - MTE. Banco de dados do GEPETIS

O grau de concentração e especialização da indústria de produtos minerais não metálicos em 1990 estava localizado em seis municípios do estado. São eles: Assú, Currais Novos, Ipanguaçu, Macaíba, Parelhas e São Gonçalo do Amarante. Em 2010, esse número de municípios especializados cresce significativamente (Figura 2).

Figura 2. Localização do emprego industrial no Rio Grande do Norte 2010

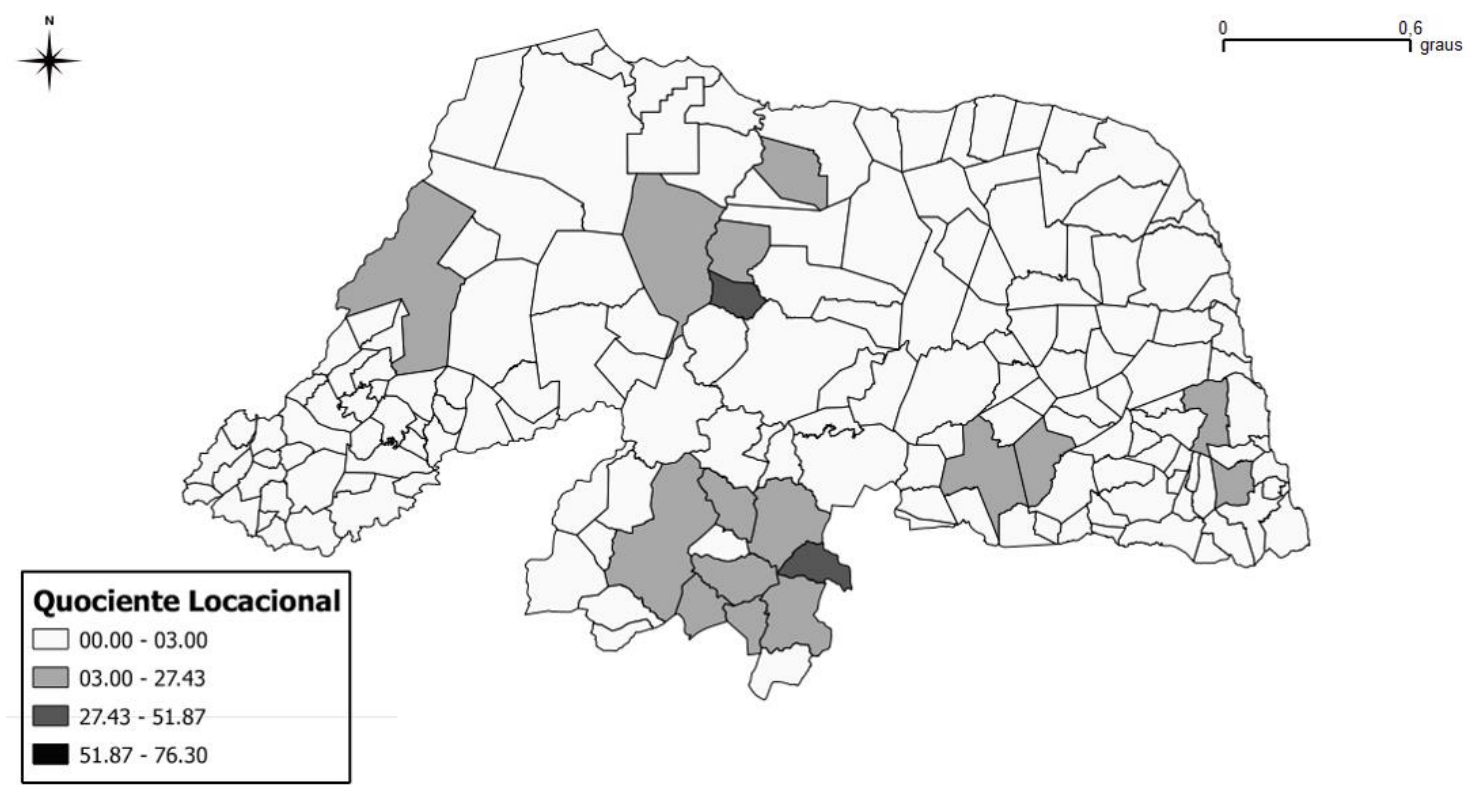

Fonte: Elaboração própria a partir dos dados da RAIS - MTE. Banco de dados do GEPETIS 


\section{CONSIDERAÇÕES FINAIS}

0 processo de desconcentração industrial e gerencial das empresas durante a década de 90 provocou uma necessidade de novas firmas se instalarem em outras regiões. Tendo em vista que grande parte das empresas estavam localizadas na Região Sudeste, com o processo de desconcentração industrial, estas empresas começam a se instalar em outras localidades, provocando assim um maior desenvolvimento de outras regiões. Isso é de grande importância para a Região Nordeste, e no contexto deste trabalho, para os estados da Paraíba e Rio Grande do Norte, que vêm ganhando com isso, pois novas firmas estão sendo instaladas nestes estados. Há assim, uma tendência de grande melhoria no mercado de trabalho, levando em consideração o emprego do trabalho de um maior número de pessoas e o consequente aumento da qualidade de vida da população.

Conclui-se por meio dos dados coletados da Relação Anual de Informações Sociais (RAIS) e por meio da metodologia utilizada por Paixão (2012) a qual se denomina índice de Hoover, que o setor industrial no Rio Grande do Norte concentra um forte nível de especialização. 0 setor da indústria de produtos minerais não metálicos, apresentando expressivo aumento de concentração e a partir disso uma maior possibilidade de força para modificação do espaço no qual é desenvolvido.

Sendo assim, é perceptível a importância que este setor deve possuir no momento de elaboração de planos de infraestrutura, os quais poderiam fomentar ainda o desenvolvimento da atividade industrial de produção mineral não metálicos. 0 mapeamento das aglomerações da indústria de produtos minerais não-metálicos, mostrou que algumas cidades do Rio Grande do Norte, vêm com um alto índice de especialização em tal ramo industrial, como o município de Itajá por exemplo.

\section{REFERÊNCIAS}

BRASIL. Ministério do Trabalho e do Emprego (MTE). Relação Anual de

Informações Sociais (RAIS). Brasília - DF; MTE. Instituto Brasileiro de Geografia e Estatística (IBGE).

Disponível em: www.ibge.gov.br

FERNANDES, A. C.; LIMA, J. P. R. Cluster de serviços: contribuições conceituais com base em evidências do pólo médico do Recife. Revista Nova economia. vol.16 no.1 Belo

Horizonte Jan./Apr. 2006. Disponível em: http://www.scielo.br/scielo.php?script=sci_arttext \&pid=S0103-63512006000100001 Acesso em: 03/10/2019.

LARA, Fernando Maccari. Notas sobre medidas de concentração e especialização: um exercício preliminar para o emprego no Rio Grande do Sul.
Secretaria do Planejamento e gestão, Fundação de Economia e Estatística, Porto alegre, outubro 2010. LOPES NETO, Alfredo. O que é Cluster? Revisão Bibliográfica, Workshop em Chihuahua - México e iniciativa pelo Nordeste. Edições Iplance. Fortaleza, 1998

PEREIRA, William E. N. Reestruturação do Setor Industrial e Transformação do Espaço Urbano de Campina Grande - PB a partir dos anos 1990. Tese de doutoramento em Ciências Sociais. CCHLA. UFRN, 2008.

Portal do Governo do Rio Grande do Norte.

Disponível em:

http://www.rn.gov.br/Conteudo.asp?TRAN=ITEM\& $\mathrm{TARG}=12083 \& \mathrm{ACT}=$ null $\& \mathrm{PAGE}=0 \& \mathrm{PARM}=$ null $\& \mathrm{LB}$ $\mathrm{L}=$ Conhe $\% \mathrm{C} 3 \% \mathrm{~A} 7 \mathrm{a}+\mathrm{o}+\mathrm{RN}$ 
REZENDE, Autenir C. Clusterização e Localização da Indústria de Transformação no Brasil entre 1994 e 2009. Encontro Regional de Economia. Fortaleza.

2012.

RODRIGUES, Marcos Aurélio; et. al. Identificação e análise Espacial das Aglomerações Produtivas do Setor de Confecções na Região Sul. Economia Aplicada, v. 16, n. 2, 2012, pp. 311-338.

SILVEIRA NETO, R. M. Concentração Industrial

Regional, Especialização

Geográfica e Geografia Econômica. Revista

Econômica do Nordeste, Fortaleza, v.36, n.2, p. 189-

208, abr.-jun. 2005.
SOARES, Francisco de Assis. Interiorização e Reestruturação da Indústria do Ceará no Final do Século XX. Revista Econômica do Nordeste, Fortaleza, 2007.

TORRES, André C. B. Alves. A caracterização do Cluster de melão de Mossoró-Baraúna RN. Programa de Pós-Graduação em Economia. João Pessoa, 2002. 